\title{
Tagungsberichte
}

\section{DIOXIN 2004}

\section{4th International Symposium on Halogenated Environmental Organic Pollutants and POPs 6. bis 10. September 2004 in Berlin}

\section{Marianne Rappolder}

Umweltbundesamt, FG IV 2.4 Ökotoxikologische Bewertung, Postfach 3300 22, D-14191 Berlin (marianne.rappolder@uba.de)

Dioxinexperten aus der ganzen Welt treffen sich jährlich zu einem einwöchigen internationalen Symposium, um die neuesten Erkenntnisse und Forschungsergebnisse über halogenierte organische Schadstoffe vorzustellen und zu diskutieren. In diesem Jahr fand der Kongress mit über 1000 Teilnehmern aus 40 Ländern an der TU Berlin statt. Dioxine und dioxinähnliche PCB spielen zwar weiterhin eine zentrale Rolle, jedoch nehmen andere persistente Stoffe, insbesondere bromierte Flammschutzmittel, aber auch perfluorierte Verbindungen, einen immer breiteren Raum ein.

WOLFGANG RoTARD (TU Berlin, Symposium-Chair) eröffnete den Kongress. Nach den Grußworten vom Vizepräsidenten der TU, Jörg STEINBACH, sprach Staatssekretär UwE LAHL vom Bundesumweltministerium über den Stand der Minderung von Dioxinen in Deutschland. Anhand des Rückgangs der Dioxinkonzentration in der Luft und in der Muttermilch erläuterte er den Erfolg der regulatorischen Maßnahmen. Außerdem präsentierte er einen vom Bundesumweltministerium erarbeiteten Vorschlag für Zielwerte für Dioxine und dioxinähnliche PCB in Lebensmitteln. Bei erfolgreicher Umsetzung dieser Zielwerte durch weitere Reduzierungsmaßnahmen würde die Mehrheit der Bevölkerung die tolerierbare tägliche Aufnahme von 2 pg WHO$\mathrm{TEQ} / \mathrm{kg}$ Körpergewicht unterschreiten. Die europäische Kommission will bis Ende 2004 Zielwerte für Futtermittel und Lebensmittel für die europäische Bevölkerung festlegen.

Der Direktor des UN-Umweltprogramms, KLAUS TÖPFER, sprach in seinem einführenden Plenarvortrag über die Ziele der POPKonvention (Stockholmer Übereinkommen über persistente organische Schadstoffe) sowie deren Zusammenwirken mit der Rotterdam-Konvention (über den Handel mit POPs) und der Baseler-Konvention (über die Abfallbeseitigung der POPs). Er erinnerte daran, dass die in den Konventionen beschlossenen Aufgaben zur Eliminierung der POPs (z.B. Nationaler Minderungsplan, Inventare) auch für die Länder gelten, die schon weitreichende Minderungsmaßnahmen durchgeführt haben.

Neben drei weiteren einführenden Plenarvorträgen fanden ca. 290 Vorträge in fünf parallelen Sitzungen statt. Annähernd 400 Poster wurden präsentiert. Aus der Fülle der Informationen können hier nur exemplarisch einige Ergebnisse dargestellt werden. Das vollständige Programm kann über das Internet $<$ http://www.dioxin2004.org > eingesehen und einschließlich der Veröffentlichungen abgeladen werden.

Schwerpunktthemen des Symposiums waren (in Klammern Anzahl der Präsentationen)

- Analytik (137)

- Entstehung, Quellen, Dekontamination (72)

- Umwelt: Konzentrationen und Trends, Transport, Verbleib und Effekte (135)

- Konzentrationen und Trends in Futter- und Lebensmitteln (41)

- Bromierte Verbindungen - Analytik, Konzentrationen, Trends, Effekte (47)

- Perfluorierte Verbindungen (18)

- Exposition des Menschen (77)

- Toxikologie (78)

- Risikobewertung und Risikomanagement (60)

\section{Analytik}

Fortschritte in der Analytik ergeben sich durch verbesserte Techniken bei der Probenahme (Passivsammler) und bei der Probenaufarbeitung (Mikrowelle, Clean up für mehrere POPs, kleinere Probenmengen für Humanproben). Durch optimierte GC-Analytik konnte zum einen eine bessere Empfindlichkeit und Selektivität erreicht werden, zum anderen können mehrere Substanzgruppen gleichzeitig in einer Probe bestimmt werden, z.B. Dioxine (PCDD/F) und polybromierte Diphenylether (PBDE). Neben der etablierten GC-HRMS (hochauflösendes Massenspektrometer) wurden als gleichwertige und preiswertere $\mathrm{Al}$ ternativen Ion trap MS und GCxGC-MS vorgestellt. Enantioselektive Analysen der PCB erlauben Rückschlüsse auf die Quellen, die Akkumulation und auf den Metabolismus.

\section{Entstehung, Quellen, Dekontamination}

Viele Präsentationen zeigten neue Messergebnisse zu den bekannten Quellen. Untersuchungen in Müllverbrennungsanlagen lassen vermuten, dass nicht nur Kupfer, sondern auch Chrom ein Katalysator für die Entstehung von Dioxinen sein kann. Als möglicherweise unterschätzte Quellen gelten neben Ablagerungen auf Mülldeponien, Asche und Schlacken auch Textilfarben und Trocknungsprozesse. Zementöfen scheinen auch bei einer Beimengung von Sondermüll als Heizmaterial den Emissionsgrenzwert von $0,1 \mathrm{ng} \mathrm{TEQ} /$ $\mathrm{Nm}^{3}$ einzuhalten. Dies ergab eine weltweite Auswertung von Emissionsmessungen bei mehr als 1700 Zementöfen [1].

PCB-Dichtungsmassen in Gebäuden aus Betonplatten (innen und außen), die zwischen 1955 und 1980 errichtet wurden, stellen eine spezifische Emissionsquelle dar. In der Schweiz wurden deshalb 1348 Dichtungen und 130 Innenraumproben bei diesen Gebäuden untersucht [2]. Knapp die Hälfte der Proben enthielten PCB-Gehalte über $50 \mathrm{mg} / \mathrm{kg}$. Die Messungen in der Innenraumluft ergaben bei einem Viertel der Proben erhöhte Konzentrationen über $>1 \mu \mathrm{g} / \mathrm{m}^{3}$. Man schätzt, dass in der Schweiz insgesamt 50 bis $150 \mathrm{t}$ $\mathrm{PCB}$ in Dichtungsmassen von Gebäuden verwendet wurden. In Schweden geht man von 150 bis 600 t PCB in derzeit noch stehenden Gebäuden aus. Die vorhandenen PCB-haltigen Dichtungsmassen sind in der Schweiz - und sicherlich auch in den übrigen europäischen Ländern - eine signifikante, diffuse PCB-Quelle.

\section{Umwelt: Konzentrationen und Trends, Transport, Verbleib und Etfekte}

Zahlreiche Präsentationen zeigten Daten aus vielen Ländern zu Konzentrationen und Trends in Böden, Sediment, Wasser, Klärschlamm, in Pflanzen und Tieren. Neben Dioxinen und PCB wurden PDBE und weitere halogenorganische Verbindungen analysiert. Dabei ist zu beobachten, dass immer häufiger Zeitreihen mit archiviertem Probenmaterial erstellt werden. Der große Vorteil liegt darin, dass neue, moderne Verfahren zur Analytik 'alter' Proben eingesetzt werden können. Probenbanken erweisen sich daher als unverzichtbare Hilfe für retrospektive Zeitreihen von neu aufkommenden Schadstoffen. 
JOHANSSON [3] präsentierte Ergebnisse zu halogenorganischen Schadstoffen aus dem französischen Muschelmonitoring-Programm. Die Muscheln werden im Mündungsgebiet der Seine seit 19:82 gesammelt und archiviert. Während bis 1997 ein Rückgang der PCB-Konzentrationen und der Belastung mit Chlorpestiziden festgestellt werden konnte, stieg der Gehalt einzelner bromierter Flammschutzmittel in dieser Zeit stark an. Erhöhte Konzentrationen von PCB, DDT und PBDE von 1997 bis 2001 sind vermutlich auf das Hochwasser in diesen Jahren zurückzuführen. 2002 zeigten diese Schadstoffe wieder ein sehr viel niedrigeres Niveau.

Brassen aus deutschen Flüssen, archiviert in der deutschen Umweltprobenbank, zeigen Dioxingehalte, die teilweise über dem EU-Höchstgehalt für Fische von $4 \mathrm{pg} / \mathrm{kg}$ Frischgewicht liegen. Die Auswertung der Proben nach dem Elbe-Hochwasser 2002 ergab, dass vor allem das Überschwemmungsgebiet der Mulde eine erhöhte Dioxinbelastung in Fischen verursachte [4].

Zahlreiche Untersuchungen von Fischen aus verschiedenen Regionen ergaben ein sehr unterschiedliches Bild der Belastung. So wurden in mehreren Präsentationen Lachse von Zuchtfarmen mit wilden Lachsen verglichen. Die Ergebnisse waren hierbei widersprüchlich und bedürfen weiterer Überprüfungen.

Eine unmittelbare Vergleichbarkeit der präsentierten Daten war z.T. dadurch erschwert, dass die Ergebnisse mit unterschiedlicher Bezugsbasis wiedergegeben wurden (Fett, Trockengewicht, Frischgewicht usw.) oder teilweise mit unterschiedlichen TEFKonzepten berechnet wurden (I-TEF, WHO-TEF für Säugetier, Vogel, Fisch). Daher sollten Veröffentlichungen alle notwendigen Informationen enthalten, die eine Umrechnung und damit auch bessere Vergleichbarkeit der Ergebnisse ermöglichen.

\section{Konzentrationen und Trends in Futter- und Lebensmitteln}

In mehreren Präsentationen wurden Ergebnisse zu Futter- und Lebensmitteln vorgestellt. Untersuchungen von Butterproben [5] und die Ergebnisse von JoA et al. [6] zeigen, dass die Dioxin- und PCB-Belastungen in den neuen Mitgliedstaaten der europäischen Union in unbelasteten Gegenden nicht höher als im restlichen Europa liegen.

Der Transfer von Dioxinen und PCB über Grasfutter in die Milch wurde von KERST et al. [7] untersucht. Der Gehalt an dioxinähnlichen PCB trägt in den untersuchten Gras- und Kuhmilchproben mit etwa $80 \%$ zum Gesamt-TEQ (Dioxine und PCB) bei. Bezogen auf den TEQ findet sich etwa die Hälfte der im Gras vorhandenen Dioxine in der Kuhmilch wieder. Beim PCB-TEQ liegt die mittlere Carry over-Rate etwas niedriger.

\section{Bromierte Flammschutzmittel - Analytik, Konzentrationen, Trends, Effekte}

In der arktischen Umwelt konnten in der Luft, in Böden und Sedimenten sowie in Tieren PBDE-Kontaminationen nachgewiesen werden. In der Muttermilch stieg die Konzentration seit 1990 um das Dreifache. Der Nachweis von dekabromiertem Diphenylether (BDE 209) in der Arktis beweist, dass auch bei diesem Kongener ein weitreichender Transport angenommen werden muss [8].

Die Expositionsdaten bromierter Flammschutzmittel zeigen um 10 - bis 100-fach höhere Gehalte in den USA gegenüber dem europäischen Festland. VIETH [9] präsentierte die Ergebnisse einer Muttermilchstudie in Deutschland, bei der 143 Proben untersucht wurden. Die Summe von 9 PBDE lag im Mittel bei 2,2 ng/g Fett. Vegetarierinnen wiesen eine niedrigere Konzentration in der Muttermilch auf. Diskutiert wurde hier als Ursache neben der Ernährung auch der Einfluss eines anderen Lebensstils.
MUIR [10] beschreibt, dass in den USA und in Kanada immer häufiger Schilddrüsenunterfunktionen bei Kindern und Störungen bei der neuronalen Entwicklung zu beobachten sind, die sich in Lern- und Verhaltensstörungen äußern. PCB, DDT, aber auch PBDE, können solche Effekte verursachen. Die PBDE-Konzentration in der Muttermilch verdoppelt sich in den USA etwa alle 3 Jahre. Geht man davon aus, dass der Wirkungsmechanismus der PBDE dem der PCB ähnelt, so kann auch hier eine Konzentration von $1250 \mathrm{ng} / \mathrm{g}$ Fett (Muttermilch) als kritisch angesehen werden. Bei einer weiterhin so schnell steigenden Exposition könnten große Teile der Bevölkerung innerhalb weniger Jahre diese kritische Konzentration erreichen. Stimmt die Annahme einer additiven Wirkung von PBDE mit PCB und DDT und berücksichtigt man die bereits vorhandenen Belastungen mit diesen Stoffen, so liegen schon jetzt Teile der Bevölkerung in den USA über diesem kritischen Wert.

Forschungsbedarf besteht vor allem für dekabromierte Diphenylether (BDE 209), die analytisch schwierig zu bestimmen sind und für hyroxylierte Metaboliten, die bis jetzt kaum untersucht wurden.

\section{Perfluorierte Verbindungen}

Die Analytik zur Bestimmung perfluorierter Verbindungen stellt eine große Herausforderung dar, da diese Verbindungen neben vielen Produkten auch in der herkömmlichen Laborausrüstung zu finden sind. Viele Präsentationen beschäftigten sich mit den Nachweisverfahren, die sich in den letzten Jahren rasch entwickelten. Die bisher durchgeführten Untersuchungen zeigen, dass diese Stoffgruppe weltweit in hohen Konzentrationen in der Umwelt nachweisbar ist. Perfluorierte Verbindungen unterscheiden sich in ihren Eigenschaften von den klassischen POPs, z.B. binden sie an Proteine. Ihre Konzentration wird daher nicht im Fettanteil des Blutes, sondern im Vollblut oder Serum bestimmt. Blutuntersuchungen aus Schweden lassen vermuten, dass perfluorierte Stoffe in sehr hoher Konzentration im Blut zu finden sind und sich die Werte weder alters- noch geschlechtsabhängig unterscheiden [11].

\section{Exposition des Menschen}

Präsentationen zur Exposition des Menschen zeigen unterschiedliche geographische Belastungssituationen bei den verschiedenen Schadstoffgruppen. In Australien sind Gehalte von Dioxinen und dioxinähnlichen PCB in der Muttermilch und im Blut sehr viel niedriger als in der nördlichen Hemisphäre [12]. WITTSIEPE et al. [13] untersuchten Blut und Muttermilch von 169 Müttern in Nordrhein-Westfalen. Die Dioxin- und PCB-Konzentrationen von Blut und Muttermilch sind auf Fettgehalt bezogen vergleichbar; allerdings unterscheidet sich die Kongenerenzusammensetzung geringfügig. In der Muttermilch ist z.B. der Gehalt an OCDD niedriger und an PCB 126 höher. Außerdem stieg der TEQ mit dem Alter der Mütter an.

\section{Toxikologie}

Die Präsentationen zu toxikologischen Wirkungen durch Dioxine, PCB, bromierte Flammschutzmittel und andere persistente Stoffe zeigten, dass multiple Effekte in den einzelnen Spezies zu beobachten sind. Die einzelnen Stoffgruppen haben meist ein jeweils eigenes toxikologisches Profil, das mit unterschiedlichen Wirkungsmechanismen zu teilweise ähnlichen Effekten führt.

In epidemiologischen Studien von Kohorten, die besonders mit Dioxinen und PCB belastet sind, können unterschiedliche Effekte beobachtet werden. AMIRova [14] stellte Daten von Arbeitern in Russland vor. Hier kam es zu signifikanten Geschlechtsver- 
schiebungen in den nachfolgenden Generationen. So wurde die Geburt von mehr Mädchen bei belasteten Vätern und mehr Jungen bei der 3. Generation von belasteten Großvätern und deren männlichen Nachkommen beobachtet. Australische Vietnam-Veteranen erkrankten vermehrt an bestimmten Krebsarten, z.B. Prostatakarzinom, Lungenkarzinom, Krebs im Kopf und Halsbereich sowie Hodgkin [15].

In einer japanischen Mutter-Kind-Studie konnten bei höheren Konzentrationen von chlororganischen Kontaminanten geschlechtsspezifische Effekte wie veränderte Hormonspiegel und Zusammensetzung der Lymphozythen [16] sowie unterschiedliche Einflüsse auf motorische, soziale und sprachliche Fähigkeiten nachgewiesen werden [17].

\section{Risikobewertung und Risikomanagement}

In einer gesonderten Sitzung wurde über das TEF-Konzept diskutiert. Dioxine und dioxinähnliche PCB werden mit Toxizitätsäquivalentfaktoren (TEF) entsprechend ihrer relativen Toxizität zu 2,3,7,8 TCDD multipliziert und anschließend als Toxizitätsäquivalente (TEQ) addiert. Man war sich einig, dass sich das TEF-Konzept bewährt hat. Mit diesem Verfahren können Mischungen von Substanzen unter Berücksichtigung ihrer relativen Potenz (REP) durch Dosis-Addition gemeinsam bewerter werden. Die Toxizitätsäquivalentfaktoren wurden immer wieder neueren Forschungsergebrissen angepasst, zuletzt 1997 auf einer Expertentagung der WHO, auf der auch Faktoren für 12 dioxinähnliche PCB festgelegt wurden. Auf dem Kongress stellte Haws [18] eine Datenbank vor, in der verfügbare Studien nach einer Qualitätssicherung mit bestimmten Kriterien aufgenommen werden können. Etwa $50 \%$ der Studien erfüllten die Kriterien und sind jetzt qualitätsgesichert mit ihren REP-Werten in dieser Datenbank enthalten. Eine erste Auswertung und der Vergleich mit den zur Zeit angewendeten Toxizitätsäquivalentfaktoren zeigen, dass einzelne Dioxin- und Furankongenere möglicherweise zu hoch eingestuft sind, während die dioxinähnlichen PCB eher zu niedrige Faktoren aufweisen [19]. Eine Aktualisierung wird daher in den nächsten Jahren angestrebt.

VERSTRAETE [20] von der europäischen Kommission erläuterte die in der EU diskutierten Vorschläge für die Verfahrensweise der Festsetzung von Höchstgehalten und Auslösewerten von Dioxinen und dioxinähnlichen PCB in Lebensmitteln und Futtermitteln. Die seit dem 1. Juli 2002 gültigen Höchstmengen gelten nur für Dioxine und Furane. Ab 1. Januar 2005 sollen die dioxinähnlichen PCB mit eingeschlossen werden. Basis sind die in der EU ermittelten Dioxin- und PCB-Gehalte in Lebensmitteln. MaLISCH [21] stellte die Ableitung der bisherigen Dioxinhöchstgehalte und Auslösewerte vor. Sie wurden aus den in der EU berichteten Hintergrundwerten etwa im Bereich des 95. Perzentils bis über den Maximalwert festgesetzt. Ziel ist dabei, die Verbraucher vor extrem hoch belasteten Lebensmitteln zu schützen. Durch die Anbindung der Höchstwerte an die vorgefundene Belastung, und nicht an das festgestellte Risiko, soll die Marktfähigkeit der Produkte weitestgehend erhalten bleiben. Toxikologische Aspekte spielen bei der Festsetzung kaum eine Rolle. Milchuntersuchungen in Deutschland, Frankreich und Spanien ergeben etwa gleich hohe Gehalte an Dioxinen und dioxinähnlichen PCB. Jedoch ist die Definition, bis zu welcher Höhe ein Ergebnis als Hintergrundwert gilt, nicht eindeutig definiert und wird von den Ländern unterschiedlich gesehen. Dies kann sich letztendlich auf die Festsetzung der Höchstgehalte auswirken. Vom deutschen Umweltbundesamt wurden Kriterien für die Risikobewertung von Dioxinen und dioxin-ähnlichen PCB vorgestellt und bisherige Bewertungen daran gemessen [22].

\section{Ausblick}

Das jährliche internationale Symposium 'Halogenated Environmental Organic Pollutants and POPs' ist die wichtigste Plattform, auf der Experten in konzentrierter Form aktuelle Forschungsergebnisse zu halogenorganischen Substanzen präsentieren und austauschen können. Neue Ansätze für die Bewertung werden diskutiert, aber auch neu erkannte Risiken werden schnell aufgegriffen und untersucht. Ein Beispiel dafür sind die perfluorierten Verbindungen. So wurde vor 2 Jahren auf dem Dioxinkongress die Problematik perfluorierter Substanzen und ihr ubiquitäres Vorkommen aufgezeigt, letztes Jahr vor allem damit verbundene analytische Probleme diskutiert, und in diesem Jahr konnten bereits die Ergebnisse mehrerer Studien vorgestellt werden. Nächstes Jahr findet zu diesem Thema der 2tägige Workshop FLUOROS (19. bis 20. August) in Toronto statt, unmittelbar vor dem 25. Dioxinkongress (Toronto, 21.-26. August 2005).

Die CD-ROM zum 24. Dioxinkongress mit allen Präsentationen (Organohalogen Compounds - Vol. 66), Informationen und umfangreichen Suchfunktionen kann über die TU Berlin Servicegesellschaft für 25 Euro (zzgl. Versandkosten) erworben werden: <kongresse@tu-servicegmbh.de>.

\section{Literatur}

[Alle hier genannten Literaturstellen sind der Tagungs-CD-ROM 'Organohalogen Compounds - Vol. 66, 2004' entnommen. Angegeben werden Autor, ID-Nummer der Präsentation und Seitenzahlen]

[1] Karstensen KH, ID-Nr. 674, 1301-1304

[2] Zennegg M, Kohler M, Tremp J, Seiler C, Minder-Kohler S, Beck M, Lienemann P, Wegmann L, Schmid P, ID-Nr. 260, 899-904

[3] Johansson I, Moisan K, Guiot N, Truquet I, Munschy C, Tronczynski J, ID-Nr. 488, 1868-1876

[4] Schröter-Kermani Ch, Herrmann Th, Päpke O, Stachel B, ID-Nr. $498,1779-1782$

[5] Malisch R, Dilara $\mathrm{P}$, ID-Nr. 345, 2080-2084

[6] Joas A, Portrykus A, Holoubek I, Umiauf G, ID-Nr.562, 2522-2528

[7] Kerst M, Waller U, Reifenhäuser W, Körner W, ID-Nr. 649, 24402444

[8] de Wit C, Alaee M, Muir D, ID-Nr. 632, 3811-3816

[9] Vieth B, Herrmann Th, Mielke H, Ostermann B, Päpke O, Rüdiger Th, ID-Nr. 319, 2643-2648

[10] Muir Th, ID-Nr. 427, 3951-3958

[11] Lindström G, Kärrman A, Van Bavel B, Hardell L, Hedlund B, IDNr. 317, 2639-2642

[12] Harden F, Mueller JF, Toms LML, Moore M, Burniston D, Symons R, Ahokas J, Fuerst P, Paepke O, ID-Nr. 445, 2847-2852

[13] Wittsiepe J, Fürst $P$, Schrey $P$, Lemm F, Kraft M, Eberwein G, Winneke G, Wilhelm M, ID-Nr 492, 2865-2872

[14] Amirova Z, Kruglov E, Dardynskaia I, ID-Nr. 183, 3202-3209

[15] Wilson E, Horsley K, van der Hoek R, ID-Nr. 410, 3677-3682

[16] Nagayama J,Tsuji H,lida T, Nakagawa R, Matsueda T, Hirakawa H, Shiraha A, Yanagawa T, Fukushige J, Watanabe T, ID-Nr. 204, $3217-3223$

[17] Nagayama J, Fukushige J, lida T, Nakagawa R, Matsueda T, Hirakawa H, Shiraha A, Yanagawa T, Watanabe T, ID-Nr. 203, 3210-3216

[18] Haws L, Harris M, Su St, Birnbaum L, Devito M, Farland W, Walker N, Connor K, Santamaria A,Finley B, ID-Nr. 599, 3426-3432

[19] Haws L, Harris M, Su St, Walker N, Birnbaum L, Devito M, Farland W, Connor K, Santamaria A, Finley B, ID-Nr. 602, 3439-3445

[20] Gallani B, Verstraete F, Boix A, von Holst Ch, Anklam E, ID-Nr. 26 , 1917-1924

[21] Malisch R, Wambold Ch, Fraisse D, Durgeil A, Defour St, Abad E, Abalos M, Rivera J, Fürst $P$, ID-Nr. 667, 2046-2052

[22] Gies A, Neumeier G, Rappolder M, Konietzka R, ID-Nr. 363, 34663471 